\title{
CME in der Aktuellen Dermatologie CME in Aktuelle Dermatologie
}

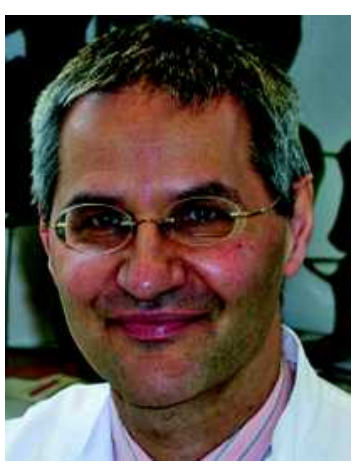

Prof. Dr. med. Detlef Zillikens
Der wissenschaftliche Fortschritt fordert von Ärzten lebenslanges Lernen: Die Halbwertszeit des medizinischen Wissens sinkt - der Informationsbedarf der Ärzte steigt.

Seit 2004 besteht deshalb in Deutschland eine Fortbildungspflicht für Fachärzte. Dabei hat sich die Fortbildung durch CME (Continuous Medical Education) als sehr effizient erwiesen: Sie deckt den steigenden Informationsbedarf und weist gleichzeitig die gesetzlich vorgeschriebene Fortbildung nach.

Aus diesem Grund haben sich Herausgeber und Verlag entschieden, auch den Leserinnen und Lesern der Aktuellen Dermatologie ein CME-Verfahren anzubieten. In dieser Ausgabe der Aktuellen Dermatologie finden Sie den ersten CMEArtikel. Weitere Arbeiten folgen. So werden strukturiert Themen aus dem gesamten Gebiet der Dermatologie aufgearbeitet, ergänzt durch Fachgebiete, in denen die Dermatologen üblicherweise Zusatzbezeichnungen erwerben müssen.

Pro Artikel können Sie 3 CME-Punkte erwerben. Natürlich ist auch eine Online-Teilnahme möglich.

Das kontinuierliche, berufsbegleitende Auffrischen und Ergänzen von Wissen hat in der Dermatologie Tradition - die neue Rubrik knüpft an diese Tradition an.

Wir hoffen, Ihnen mit dieser Fortbildungsmöglichkeit einen wertvollen Zusatznutzen bieten zu können. Übrigens nehmen wir gerne Anregungen für Themen aus dem Leserkreis entgegen - nicht zuletzt deshalb, um den Austausch mit Ihnen, liebe Leserinnen und Leser, zu fördern.

Ich würde mich freuen, wenn durch diese neue Rubrik Ihre Fortbildung noch effizienter wird und noch mehr Freude bereitet.

Prof. Dr. med. Detlef Zillikens
Hinweise zum CME-Verfahren

Den ersten Beitrag zur CME-Fortbildungsserie in der Aktuellen Dermatologie finden Sie ab Seite 209. Zur Teilnahme müssen Sie die Fragen auf den Seiten 222 und 223 beantworten und die Felder A-C auf Seite 224 ausfüllen. In Feld D tragen Sie bitte Ihre Abonnementsnummer ein (zu finden auf dem Adressaufkleber auf Ihrem Heft). Bitte beantworten Sie unbedingt auch die Fragen zur didaktisch-methodischen Evaluation (Feld F) auf Seite 225. Den Antwortbogen schicken Sie dann bitte zusammen mit einem frankierten Rückumschlag an den Georg Thieme Verlag. Sie können auch bequem online am CME-Verfahren teilnehmen unter http://cme.thieme.de. Unter „CME für Abonnenten“ wählen Sie dann die „Aktuelle Dermatologie“ aus. Alles Weitere erklärt sich dann im System selbst.
Bibliografie

DOI 10.1055/s-2008-1077464

Akt Dermatol 2008; 34; 201

(c) Georg Thieme Verlag KG .

Stuttgart · New York

ISSN 0340-2541

Korrespondenzadresse

\section{Prof. Dr. med. Detlef Zillikens}

Klinik für Dermatologie, Allergologie

und Venerologie

Universität zu Lübeck

Ratzeburger Allee 160

23538 Lübeck

detlef.zillikens@uk-sh.de 